\title{
Resection for Hepatocellular Carcinoma
}

\author{
Hariharan Ramesh \\ Lakeshore Hospital \& Research Center, Cochin, Kerala, India
}

\begin{abstract}
Hepatocellular Carcinoma (HCC) continues to present major challenges in management, which is further complicated by the presence of associated chronic liver disease. Key issues in surgical resection of HCC include the site, size, and number of lesions, the severity of the chronic liver disease, and the size of the functional liver remnant. De novo HCC in the absence of chronic liver disease can be treated by major liver resection with little risk of postoperative liver failure. Liver resection can also be used a bridge to liver transplantation as it affords the possibility of determining the pathologic grade of the tumortumor and its invasiveness, and thereby the prognosis. This review summarizes the current treatment approaches to surgical resection for HCC. (J CLIN EXP HePATOL 2014;4:S90-S96)
\end{abstract}

$\mathrm{R}$ ecommendations for treatment of HCC should be based on randomized controlled trials or meta analyses, rather than by non randomized clinical trials or observational studies. However, there is a paucity of robust evidence regarding the treatment of HCC. Interventions have not been thoroughly tested against each other. Hence the strength of evidence for most interventions is less than desirable. Therefore treatment decisions must be taken by a multi-disciplinary group which comprises hepatologists, surgeons, radiologists, interventional radiologists, pathologists, nurses, palliative care physicians, patient education specialists, and pharmacists. Treatment decisions must be made in a tumortumor board meeting.

In India, the problem is compounded by the fact that the country is large, and there is considerable variation in the expertise and technology available for the treatment of HCC. It is inconceivable that HCC shall be treated only in a few centers with state-of-the art services. While treatment guidelines must therefore be treated with

Keywords: liver cancer, surgery, hepatocellular carcinoma, liver tumor, resection

Received: 31.12.2013; Accepted: 5.7.2014

Address for correspondence: Hariharan Ramesh, Director of Surgical Gastroenterology \& Liver Transplantation, Lakeshore Hospital \& Research Center, Cochin, Kerala, India.

E-mails: hrameshoffice@gmail.com; drhramesh@gmail.com

Abbreviations: AFP: alpha-fetoprotein; AFP/TTV: AFP to tumor volume; ASA: American Society of Anesthesiologists; BCLC: barcelona clinic liver cancer; CT: computerized tomography; CTP: child-turcotte-pugh; CUSA: cavitary ultrasound suction aspirator; FDG-PET: fludeoxyglucose positron emission tomography; FLR: functioning liver remnant; HBV: hepatitis B virus; HCC: hepatocellular carcinoma; HPB: hepato-pancreato-biliary; HVPG: hepatic venous pressure gradient; MELD: model for end-stage liver disease; PEI: percutaneous ethanol injection; POLT: primary orthotopic liver transplantation; PVE: portal vein embolization; RFA: radiofrequency ablation; TACE: transarterial chemoembolization; UCSF: University of California, San Francisco

http://dx.doi.org/10.1016/j.jceh.2014.07.002 caution, the right of every patient with HCC to standardized care has placed a huge burden on healthcare systems to deliver for these patients. Although prevention of HBV related cancer is still the priority, yet, treatment of the established HCC is also necessity.

The radical treatment options are:

a) Surgical resection

b) Liver transplantation

c) Local ablative techniques such as

a. Radiofrequency ablation (RFA)

b. Percutaneous ethanol injection (PEI)

No randomized data is available at this time comparing these three approaches. Hence existing guidelines are dependent on cure rates with these treatment approaches.

\section{HEPATOCELLULAR CARCINOMA IN THE ABSENCE OF LIVER DISEASE}

HCC in background of healthy liver (without cirrhosis or chronic hepatitis) may occur occasionally. ${ }^{1}$ There are two types: a) the classical sporadic variety, and b) the fibrolamellar variant. The fibrolamellar variant is characteristic in the female preponderance, absence of AFP elevation, and in the presence of lymph node involvement. These tumors present only as mass lesions and at an advanced stage. Despite this advanced stage at presentation, resection can be done as the healthy liver has a normal ability to regenerate. Survival following resection is over $50 \%$ at 5 years. ${ }^{2}$

\section{HEPATOCELLULAR CARCINOMA IN THE PRESENCE OF LIVER DISEASE}

Resection for HCC has several advantages: a) it requires no waiting time, b) allows pathologic examination of the tumortumor and therefore prognostication, and c) atleast in theory, does not preclude future liver transplantation. In this role it is used a bridge to transplantation. 
However, liver resection is clearly inferior to liver transplantation when applicable as a long term treatment modality in HCC with liver disease as.

a) There is the potential to miss satellite lesions and nonvisible tumor

b) The remaining liver tissue continues to present a risk of developing new HCC;

c) There is a risk of deterioration of liver function either immediately following hepatic resection or later.

It is important to emphasize that liver transplantation is not applicable to all tumors (Milan, UCSF or other criteria), and liver resection is not suitable in patients with decompensated liver function. Further, liver transplantation requires the availability of cadaveric or living donors, and a specialized transplant setup. Liver resection can, on the other hand, be performed in any center where adequate surgical, medical and radiologic facilities are available. Currently there exists in India, larger numbers of successful hepato-pancreato-biliary (HPB centers) as opposed to liver transplant centers although this situation is rapidly changing. Recent meta-analysis has shown comparable survival figures in patients with early HCC treated (intention-totreat strategy) by resection or transplantation. There is also some data that primary orthotopic liver transplantation (POLT) may be more cost effective than resection with salvage transplantation in HCC in Child A cirrhotics. ${ }^{3}$

\section{RESECTION IN PATIENTS WITH UNDERLYING CHRONIC LIVER DISEASE}

\section{Assessment of the Severity of Liver Fibrosis or Cirrhosis}

Assessment of the severity of liver fibrosis or cirrhosis is crucial. Clinical pointers are a) Child-Pugh or MELD scores, b) platelet counts, c) splenomegaly and d) esophageal varices.

Large series have reported 10 year recurrence-free survivals of $22 \%$ of 22800 patients who have undergone liver resection for HCC. The results are best in patients who have a single tumortumor with a simple nodular appearance where there is no vascular invasion or intrahepatic metastases. ${ }^{4}$ Overall, the application of resection and the results following resection are governed by patient, liver and surgeon factors (Figure 1).

\section{Selection of Candidates for Resection}

For selection of candidates for resection use following criteria:

a) Rule out extrahepatic metastases. It is desirable to perform a CT of the chest prior to resection or transplantation. This is because HCC does metastasize to the lymph nodes, lungs or bone. A bone scan is not a routine requirement. FDG-PET scans have low sensitivity of 55\%; however, with increasing grade or stage, higher yields upto $100 \%$ may be available. ${ }^{5}$

b) Assessment of the extent of the hepatic disease: the location, size and number of tumors, their proximity to major vessels, and whether they are peripheral in location or central deep in the liver all influence the resectability. First the residual volume after resection must be calculated based on the imaging. In normal livers, residual volumes of as low as $20 \%$ are acceptable in tumor resections, but in patients with fibrosis or cirrhosis, higher residual volumes are necessary. Patients with Child A liver status may withstand major hepatectomies, but Child B category patients may only be subjected safely to minor resections. Tumor size alone may not be a deterrent to safe resections. Reports of over $45 \%$ five

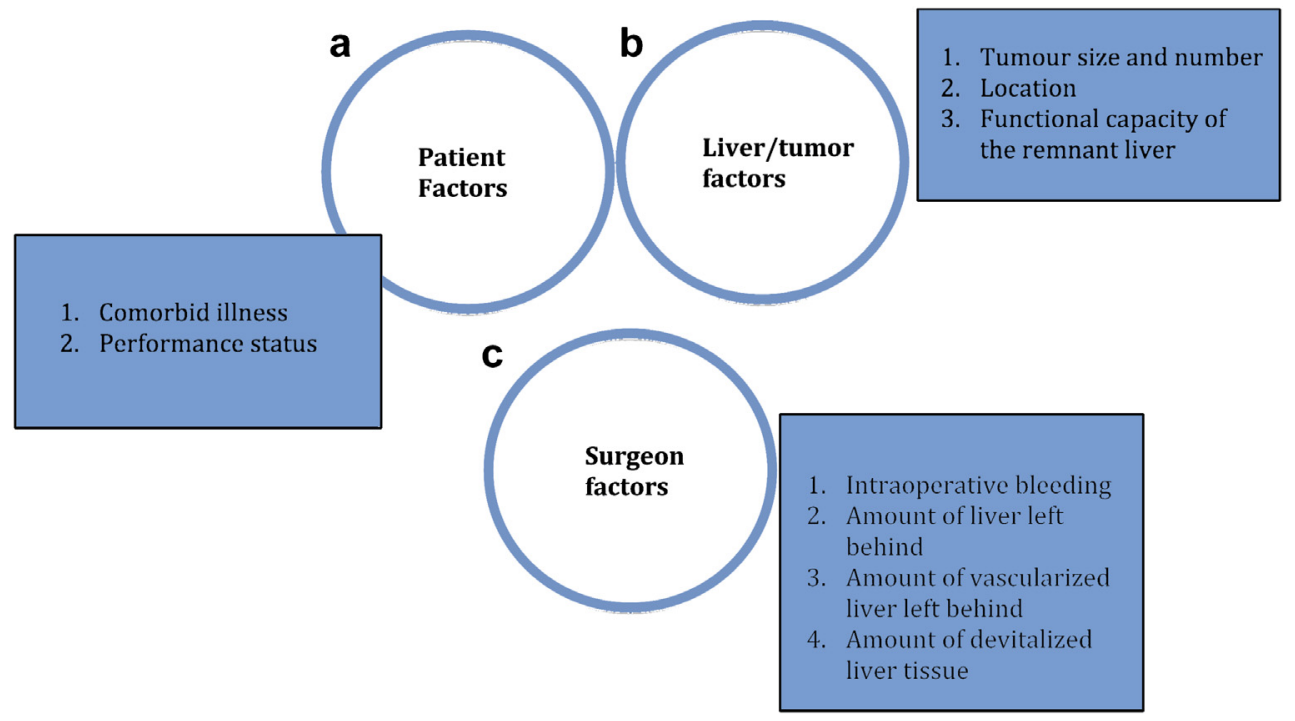

Figure 1 The results of liver resection for HCC depends on three important criteria: a) patient factors, b) liver factors, and c) surgeon factors. 
year survival in tumors larger than $10 \mathrm{~cm}$ have been reported. ${ }^{6}$ Major vascular or biliary invasion may be a contraindication, but portal vein involvement, or thrombosis within the segment of liver that is to be resected is not. ${ }^{7}$ Overall, the results among patients who have tumors with portal vein invasion are poorer than those in whom invasion is not present. ${ }^{8}$

Multinodular tumors however have poorer prognosis and recurrence rates are $80-100 \%$ although 5 year survival rates of $30 \%$ are also reported. Hence these may only be resected if transplantation is not feasible. ${ }^{9,10}$

c) Assessment of hepatic functional reserve: This involves three major tasks: a) determining the functional residual volume after resection, and b) assessment of liver function status and c) assessment of the severity of portal hypertension.

Functioning liver remnant (FLR) is now calculated using volumetry after a high resolution CT scan. Residual volumes of $30 \%$ in chronic liver disease without cirrhosis and $40 \%$ in Child A cirrhosis without portal hypertension may be acceptable for safe resection. However the concept of total functional liver volume (total liver volume-tumor volume) must be understood. It may be more relevant to determine the residual volume as a measure of the total functional liver volume as opposed to total liver volume. For e.g., in Figure 2A, the residual volume is greater as most of the resected liver is tumor, where as in Figure 2B, the residual volume is smaller, and these patients have a higher propensity to postoperative liver failure. ${ }^{11}$

Liver function status may be assessed using many criteria. The Child-Turcotte-Pugh system is still a standard method; in general, patients with CTP B or C are not candidates for any major resection and must seek alternative therapies including liver transplantation. Because of the categorical nature of many variables in the CTP system, and the subjective assessment, the MELD score has been used more recently. MELD has been shown to correlate with postoperative outcomes after resection, and also help in predicting which patients are suitable for hepatectomy. A MELD score of
$>9$ has generally been associated with higher postoperative liver failure rates although there is considerable variation across published data. ${ }^{12-14}$

Indocyanine green retention as a measure of accurate functional grading: Retention of indocyanine green at $15 \mathrm{~min}$ can be used as a significant test of liver dysfunction. The test is not routinely available in India. The test is most applicable when a small hepatectomy is considered, as it gives an accurate assessment of overall liver function and therefore best used in a Child B patient who requires a segmental resection. But it cannot be applied to patients who require a hemihepatectomy, for it gives no indication of the function of the FLR. ${ }^{15,16}$ Portal hypertension is an important variable which determines the success of surgical resection. There are direct and indirect tests to assess the presence and severity of portal hypertension. While Hepatic-Portal venous gradient (HPVG) can be regarded as the gold standard, it is invasive and it has been shown that the MELD predicted outcomes more reliably than HPVG. Further portal hypertension can be gaged by many indirect tests-the presence of varices, collaterals on cross sectional imaging, splenic size and the platelet count. ${ }^{17}$

d) Some other factors which play a role in determining outcomes are: 1) elevated creatinine (indicated by MELD), 2) chronic pulmonary disease, 3) male sex, and 4) ASA (American Society of Anesthesiologists Class) IV/V. Age greater than 70 was equivalent to 3 additional MELD points, and ASA IV added 5.5 MELD points. ${ }^{18}$

\section{Preoperative Therapy Before Hepatic Resection Portal Vein Embolization}

Although this may help increase the residual liver volume if a major liver resection becomes necessary, there is a $10-20 \%$ complication rate, and $1 \%$ of cirrhotic patients may develop accelerated portal hypertension. Both systematic reviews and prospective studies have shown improved early outcomes after right hepatectomy following portal embolization in HCC. ${ }^{19,20}$ Relative contraindications to PVE are portal invasion/occlusion in the segment to be embolized and biliary obstruction of the FLR (due to the reduced
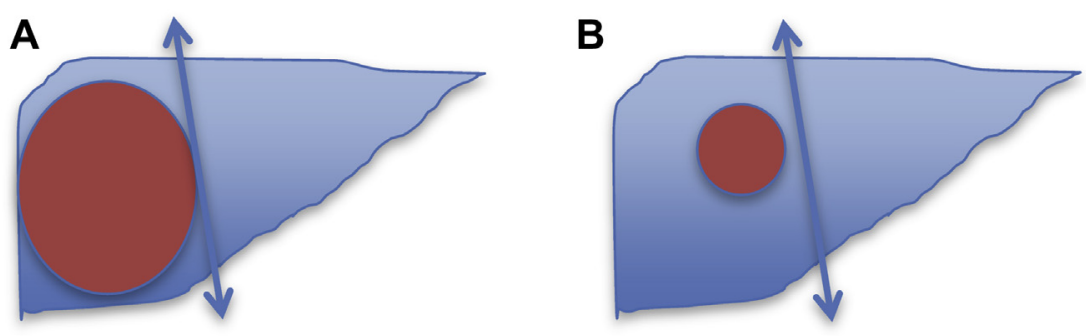

Figure 2 A: resected liver is largely tumor, and so the tumor free functional residual volume is greater, with a lower likelihood of postoperative liver failure. B: Resected liver is largely normal liver and the functional residual volume is less, and a greater likelihood of postoperative liver failure. 
ability of the obstructed liver to hypertrophy), coagulopathy, and renal failure.

\section{Transarterial Chemoembolization (TACE)}

Despite initial promise, TACE has failed to improve survival in HCC when used as a neoadjuvant therapy. Disease free and overall survival figures remained similar and further, upto $10 \%$ may not reach resection due to the detection of additional disease elsewhere in the liver. ${ }^{21}$

In some cases, a combination of PVE and TACE (applied in sequential fashion), or even hepatic vein embolization has been used ${ }^{22,23}$ because of the poor regenerative capacity of the liver in the setting of cirrhosis.

\section{PRINCIPLES OF SURGICAL RESECTION}

There has been considerable improvement in techniques of liver resection and in the results following resection. Intraoperative bleeding is a principal determinant of perioperative mortality. Previously, several retrospective studies compared the survival benefits of anatomical resection and non-anatomical resection for the treatment of HCC, because most of the previous studies encompassed patients with significantly different background with regard to AR and NAR, they did not provide concrete conclusions. At this moment, the results of these studies investigating the survival benefits of anatomical resection compared with non-anatomical resection may not be completely consistent [Tomimaru et al Dig Dis Sci 57:1942-1948].

Some salient points on principles of surgical resection are:

a) Low central venous pressure during resection. ${ }^{24}$

b) Avoid Pringle maneuver or use it as an intermittent measure. $^{25}$

c) Anatomic resections based on Couinaud segments are not essential, and care must be taken to preserve vascularized parenchyma as far as possible. ${ }^{26}$

d) While $2 \mathrm{~cm}$ margins produce greater survival than lesser margins, a $1 \mathrm{~cm}$ margin is considered adequate and will take into account the location of the tumor and its relationship to major vascular pedicles or structures. ${ }^{26-28}$

e) Liver transection techniques depend on the availability, experience and preference of the operating surgeon; Cochrane reviews have suggested that Kellyclasie is the quickest and most cost-efficient, although atypical resections may benefit from CUSA (cavitary ultrasound suction aspirator). ${ }^{29,30}$

f) Anterior approach may prove advantageous in patients with large tumors, where mobilization off the cava may involve traction on the tumor with the possibility of tumor rupture, bleeding and dissemination of tumor cells due to handling of the tumor. ${ }^{31,32}$ This may further be facilitated by the hanging maneuver where a catheter is passed by blunt dissection just anterior to the inferior vena cava and between the right and middle hepatic veins. Traction on the catheter allows a straight line of transection, and some control of bleeding in the depths in the transection. ${ }^{33,34}$ The blind dissection behind the liver and in front of the inferior vena cava required may cause bleeding from the short hepatic veins in $6-9 \%$ and necessitate abandonment of the hanging maneuver. An alternative is to hang the liver along a plane which is to the right of inferior vena cava, a technique described by Chen. ${ }^{35}$

g) Current standards describe a mortality rate of between 2 and $3 \%$, a blood transfusion rate of under $10 \%$ and with 5 year survival rates of $60 \%{ }^{36}$

\section{RESECTION VERSUS ABLATION}

Both systematic reviews ${ }^{37}$ and recent publications ${ }^{38}$ have highlighted the fact that resection produces better results than RFA especially in small HCC. However, the advantage of RFA is the lack of post-procedure adhesions which may result from surgery which provides greater technical ease in explantation during salvage transplantation. Laparoscopic liver resection has the potential to provide surgical therapy with fewer adhesions. Table 1 outlines the comparative survival data of patients who have had resection versus ablation for both tumors within and beyond the Milan criteria.

\section{LAPAROSCOPIC VERSUS OPEN RESECTION}

The first laparoscopic resections for HCC were performed in 1992, but it was not until 2000 that Daniel Cherqui from Paris published his series of 30 major liver resections. Initial reports were all of resection of anterior segments or a left lateral sectionectomy. In 2002, the first laparoscopic procurement of a liver graft had been successfully performed and by 2010, the first single incision laparoscopic resection. Laparoscopic techniques in liver resection were classified by the Louisville 2008 statement into three

Table 1 Results of RFA Versus Resection. ${ }^{37}$

\begin{tabular}{lll}
\hline & \multicolumn{1}{c}{ RFA } & Resection \\
\hline a) Within Milan criteria & & \\
\hline Median tumor size within Milan criteria & $1.8-2.1 \mathrm{~cm}$ & $2.0-2.7 \mathrm{~cm}$ \\
\hline Number of patients & 928 & 718 \\
\hline Survival 1 year (\%) & $78-83$ & $80-83$ \\
\hline Survival 3 years (\%) & $36-59$ & $49-64$ \\
\hline Survival 5 years (\%) & $17-25$ & $22-38$ \\
b) Outside Milan criteria & & \\
\hline Median tumor size outside Milan criteria & $3.0-4.6 \mathrm{~cm}$ & $4.6-7.4 \mathrm{~cm}$ \\
\hline Number of patients & 797 & 712 \\
\hline Survival 1 year (\%) & $78-98$ & $75-97$ \\
Survival 3 years (\%) & $33-94$ & $64-93$ \\
Survival 5 years (\%) & $20-75$ & $31-98$ \\
\hline
\end{tabular}


groups: a) Pure laparoscopic procedures, b) laparoscopy with a hand port, and c) hybrid procedures where the procedure is completed by a mini-laparotomy.

Laparoscopic surgery has the potential to minimize the "hit" of surgery, and consequently improve early outcomes. Laparoscopic liver resection for HCC is associated with less blood loss, shorter hospital stay, and fewer complications in selected patients with no compromise in survival. The magnification provided by laparoscopy not only facilitates meticulous hemostasis but also aids the identification of satellite nodules and this can be further aided by the use of laparoscopic ultrasound. There may be less disturbance to the collateral circulation around the liver. Other advantages of laparoscopic liver resection include: a) decrease in postoperative ascites, b) less postoperative pain, c) feasibility in some patients with cirrhosis where open surgery is contraindicated, d) fewer intra-abdominal adhesions, which may be a great help when patients are taken up for salvage transplantation, e) shorter recovery time, and f) shorter length of hospital stay. Potential disadvantages are that it requires a great deal of technical skill, especially in cases where hemihepatectomies are required. ${ }^{39}$ Gayet showed that intraoperative anatomy is best unraveled by intraoperative ultrasonography using the laparoscopic probe. ${ }^{40}$ Also, many gadgets such as vascular staplers, harmonic scalpel are required. Despite all the emerging data, recent publications still describe the majority of resections of one or two segments rather than major hepatectomies. ${ }^{41}$

Robotic techniques are also gaining momentum in liver resections for HCC. A recent report described over 200 liver resections of which $30 \%$ was for HCC. ${ }^{42}$

\section{PREDICTORS OF SURVIVAL AFTER RESECTION FOR HEPATOCELLULAR CARCINOMA}

Predictors of survival after resection for HCC are given below $^{17,41,43}$ :

a) Tumor size; Tumors less than $2 \mathrm{~cm}$ have a significantly higher survival (67\%) than those between 2 and $5 \mathrm{~cm}$ $(52 \%)$ and survival is poorer $(37 \%)$ when the tumor size exceeds $5 \mathrm{~cm}$;

b) Tumor number: Survival drops from $57 \%$ for a single nodule to as low as $26 \%$ when there are 3 or more nodules. Further, solitary nodules have significantly improved survival after resection as compared to oligonodular tumors. Fan et al reported from Hong Kong the 5 year survival of $72 \%$ among 287 younger patients with HCC and underlying liver disease treated with hepatectomy and an $\mathrm{R} 0$ resection margin, versus $81 \%$ among 50 patients who underwent right lobe liver transplantation and oligonodular tumors had the poorest prognosis. ${ }^{43}$

c) Presence of microsatellites, d) Vascular invasion, Micro vascular invasion is a very important determinant of survival and correlates well with tumor size and histological differentiation $(20 \%$ of tumors less than $2 \mathrm{~cm}$ diameter, $30-60 \%$ when the size is $2-5 \mathrm{~cm}$ and $90 \%$ when size exceeds $5 \mathrm{~cm}$ ). Survival plummets from 87 month median survival without invasion, to as low as $8-12$ months for micro vascular invasion.

e) The severity of cirrhosis, and

f) Alpha-feto protein levels. Recent data suggests that the ratio of AFP to tumor volume (AFP/TTV > 1.5) is a more accurate indicator of risk of recurrence. ${ }^{44}$ Liver resection for HCC is followed by a high recurrence rate (upto 70\%) either due to intrahepatic dissemination or de novo appearance of new lesions. Recurrences are classified as early (less than 2 years) and late (greater than 2 years). The former indicates a tumor with aggressive biology, whereas late lesions are related to the underlying cirrhosis.

\section{ADJUVANT THERAPY TO DECREASE RECURRENCES AFTER RESECTION}

To decrease recurrences after resection following adjuvant therapies may be used ${ }^{45,46}$ :

a) Alpha Interferon produces a significant improvement in recurrence-free survival (54\% vs. $30 \%$ of placebo; 9 small randomized controlled trials). However a single large RCT of 150 patients did not show a benefit, although a trend in preventing late de novo recurrence was reported. MiR-26 is a marker, which indicates response to interferon therapy. Currently interferon is not recommended as a postoperative adjuvant therapy. Interferon with $5 \mathrm{FU}$ has recently been used after resection in patients with portal vein thrombus with promising results. ${ }^{45}$

b) Internal radiation with ${ }^{131}$ I-labeled lipiodol showed some benefit.

c) Adoptive immunotherapy with activated lymphocytes with interleukin- 2 decreased recurrence rate at 3 years from $48 \%$ to $33 \%$.

d) Retinoids and Vitamin $\mathrm{K} 2{ }^{46}$ have also been tried in preventing de novo tumors.

e) New anticancer regimens including sorafenib have been reported to have a beneficial survival effect in patients with HCC. A clinical trial to examine the recurrencepreventing effect of sorafenib when administered after curative treatments such as resection or ablation (STORM trial) was recently completed.

\section{RE RESECTION OF HEPATOCELLULAR CARCINOMA}

Re-resection depends upon the underlying liver disease status, and if the liver function is stable, then re- 
resection can be done with results comparable with primary resection.

\section{RESECTION AS A BRIDGE TO LIVER TRANSPLANTATION}

Two settings have been proposed: 1) resection was used as primary therapy, and liver transplantation is performed for patients who develop recurrence and/or liver failure (rescue therapy); 2) selected patients with high recurrence risk based on resection pathology can be subjected to liver transplantation immediately even in the absence of proven recurrent disease, which is recognized as pre-planned combined therapy. ${ }^{47}$ Although earlier reports described increased mortality rates following secondary transplantation as compared to primary transplant (26.8 versus $2.1 \%$ ), most recent studies have shown comparable results. ${ }^{48}$ The principal advantage of resection is that tissue is available for a careful pathologic study which can establish the invasiveness of the tumor, and thereby predict long term outcomes after transplantation. The disadvantage stems from the postoperative adhesions which may make explantation difficult. Laparoscopic resection may be helpful in minimizing postoperative adhesions.

\section{CONCLUSIONS}

Liver resection is the treatment of choice when patients have normal livers, and in those without advanced fibrosis, provided an $\mathrm{R} 0$ resection can be carried out leaving an adequate liver remnant. In cirrhotic patients, liver resection is effective and safe in early stage HCC (BCLC 0 and A), provided that the lesion is single, the patient has a good performance status; and the portal hypertension is not severe. In multifocal tumors in patients unsuitable for liver transplantation, and mild portal hypertension, liver resection can be carried out, but requires prospective comparison with other loco-regional treatments. Liver resection can be carried out in this setting with mortality rates less than $5 \%$ and survival of $50-60 \%$. Neoadjuvant or adjuvant therapies have not improved outcomes of patients treated with liver resection. Re-resection can be carried out in selected patients with recurrence after liver resection provided liver function is stable, and R0 resection can be achieved. Salvage transplantation can be done following liver resection in HCC.

\section{CONFLICTS OF INTEREST}

The author has none to declare.

\section{REFERENCES}

1. Grando-Lemaire V, Guettier C, Chevret S, Beaugrand M, Trinchet JC. Hepatocellular carcinoma without cirrhosis in the West: epidemiological factors and histopathology of the non- tumorous liver. Groupe d'Etude et de Traitement du Carcinome Hepatocellulaire. J Hepatol. 1999;31(3):508-513.

2. Belghiti J, Kianmanesh R. Surgical treatment of hepatocellular carcinoma. HPB (Oxford). 2005;7(1):42-49.

3. Landman MP, Feurer ID, Pinson CW, Moore DE. Which is more costeffective under the MELD system: primary liver transplantation, or salvage transplantation after hepatic resection or after locoregional therapy for hepatocellular carcinoma within Milan criteria? HPB (Oxford). 2011;13(11):783-791.

4. Eguchi S, Kanematsu T, Arii S, et al. Recurrence-free survival more than 10 years after liver resection for hepatocellular carcinoma. $\mathrm{Br}$ J Surg. 2011;98(4):552-557.

5. Wolfort RM, Papillion PW, Turnage RH, Lillien DL, Ramaswamy MR, Zibari GB. Role of FDG-PET in the evaluation and staging of hepatocellular carcinoma with comparison of tumor size, AFP level, and histologic grade. Int Surg. 2010;95(1):67-75.

6. Young AL, Malik HZ, bu-Hilal M, et al. Large hepatocellular carcinoma: time to stop preoperative biopsy. J Am Coll Surg. 2007;205(3):453-462.

7. Ikai I, Yamamoto Y, Yamamoto N, et al. Results of hepatic resection for hepatocellular carcinoma invading major portal and/or hepatic veins. Surg Oncol Clin N Am. 2003;12(1):65-75. ix.

8. Pawlik TM, Poon RT, Abdalla EK, et al. Hepatectomy for hepatocellular carcinoma with major portal or hepatic vein invasion: results of a multicenter study. Surgery. 2005;137(4):403-410.

9. Wang BW, Mok KT, Liu SI, et al. Is hepatectomy beneficial in the treatment of multinodular hepatocellular carcinoma? J Formos Med Assoc. 2008;107(8):616-626.

10. Ng KK, Vauthey JN, Pawlik TM, et al. Is hepatic resection for large or multinodular hepatocellular carcinoma justified? Results from a multi-institutional database. Ann Surg Oncol. 2005;12(5):364373.

11. Schindl MJ, Redhead DN, Fearon KC, Garden OJ, Wigmore SJ. The value of residual liver volume as a predictor of hepatic dysfunction and infection after major liver resection. Gut. 2005;54(2):289296.

12. Teh SH, Christein J, Donohue J, et al. Hepatic resection of hepatocellular carcinoma in patients with cirrhosis: Model of End-Stage Liver Disease (MELD) score predicts perioperative mortality. J Gastrointest Surg. 2005;9(9):1207-1215.

13. Hsu KY, Chau GY, Lui WY, Tsay SH, King KL, Wu CW. Predicting morbidity and mortality after hepatic resection in patients with hepatocellular carcinoma: the role of Model for End-Stage Liver Disease score. World J Surg. 2009;33(11):2412-2419.

14. Cucchetti A, Ercolani G, Vivarelli M, et al. Impact of model for endstage liver disease (MELD) score on prognosis after hepatectomy for hepatocellular carcinoma on cirrhosis. Liver Transpl. 2006;12(6):966-971.

15. Lau H, Man K, Fan ST, Yu WC, Lo CM, Wong J. Evaluation of preoperative hepatic function in patients with hepatocellular carcinoma undergoing hepatectomy. Br J Surg. 1997;84(9):1255-1259.

16. Lam CM, Fan ST, Lo CM, Wong J. Major hepatectomy for hepatocellular carcinoma in patients with an unsatisfactory indocyanine green clearance test. Br J Surg. 1999;86(8):1012-1017.

17. Earl TM, Chapman WC. Conventional surgical treatment of hepatocellular carcinoma. Clin Liver Dis. 2011;15(2):353-370.

18. Teh SH, Nagorney DM, Stevens SR, et al. Risk factors for mortality after surgery in patients with cirrhosis. Gastroenterology. 2007;132(4):1261-1269.

19. Farges O, Belghiti J, Kianmanesh R, et al. Portal vein embolization before right hepatectomy: prospective clinical trial. Ann Surg. 2003;237(2):208-217.

20. Abulkhir A, Limongelli P, Healey AJ, et al. Preoperative portal vein embolization for major liver resection: a meta-analysis. Ann Surg. 2008;247(1):49-57. 
21. Chua TC, Liauw W, Saxena A, et al. Systematic review of neoadjuvant transarterial chemoembolization for resectable hepatocellular carcinoma. Liver Int. 2010;30(2):166-174.

22. Hwang S, Lee SG, Ko GY, et al. Sequential preoperative ipsilateral hepatic vein embolization after portal vein embolization to induce further liver regeneration in patients with hepatobiliary malignancy. Ann Surg. 2009;249(4):608-616.

23. Yoo H, Kim JH, Ko GY, et al. Sequential transcatheter arterial chemoembolization and portal vein embolization versus portal vein embolization only before major hepatectomy for patients with hepatocellular carcinoma. Ann Surg Oncol. 2011;18(5):1251-1257.

24. Chen H, Merchant NB, Didolkar MS. Hepatic resection using intermittent vascular inflow occlusion and low central venous pressure anesthesia improves morbidity and mortality. J Gastrointest Surg. 2000;4(2):162-167.

25. Man K, Fan ST, Ng IO, Lo CM, Liu CL, Wong J. Prospective evaluation of Pringle maneuver in hepatectomy for liver tumors by a randomized study. Ann Surg. 1997;226(6):704-711.

26. Dahiya D, Wu TJ, Lee CF, Chan KM, Lee WC, Chen MF. Minor versus major hepatic resection for small hepatocellular carcinoma (HCC) in cirrhotic patients: a 20-year experience. Surgery. 2010;147(5):676-685.

27. Shi M, Guo RP, Lin XJ, et al. Partial hepatectomy with wide versus narrow resection margin for solitary hepatocellular carcinoma: a prospective randomized trial. Ann Surg. 2007;245(1):36-43.

28. Poon RT, Fan ST, Ng IO, Wong J. Significance of resection margin in hepatectomy for hepatocellular carcinoma: a critical reappraisal. Ann Surg. 2000;231(4):544-551.

29. Pamecha V, Gurusamy KS, Sharma D, Davidson BR. Techniques for liver parenchymal transection: a meta-analysis of randomized controlled trials. HPB (Oxford). 2009;11(4):275-281.

30. Gurusamy KS, Pamecha V, Sharma D, Davidson BR. Techniques for liver parenchymal transection in liver resection. Cochrane Database Syst Rev. 2009;(1):CD006880.

31. Liu CL, Fan ST, Cheung ST, Lo CM, Ng IO, Wong J. Anterior approach versus conventional approach right hepatic resection for large hepatocellular carcinoma: a prospective randomized controlled study. Ann Surg. 2006;244(2):194-203.

32. Lai EC, Fan ST, Lo CM, Chu KM, Liu CL. Anterior approach for difficult major right hepatectomy. World J Surg. 1996;20(3):314-317.

33. Wu TJ, Wang F, Lin YS, Chan KM, Yu MC, Lee WC. Right hepatectomy by the anterior method with liver hanging versus conventional approach for large hepatocellular carcinomas. $\mathrm{Br} J$ Surg. 2010;97(7):1070-1078.

34. Belghiti J. Editorial perspective: resection of large hepatocellular carcinoma using combination of liver hanging maneuver and anterior approach. World J Surg. 2010;34(8):1879-1880.
35. Chen XP, Zhang WG, Lau WY, Qiu FZ. Right hepatectomy using the liver double-hanging maneuver through the retrohepatic avascular tunnel on the right of the inferior vena cava. Surgery. 2008;144(5):830-833.

36. Jarnagin W, Chapman WC, Curley S, et al. Surgical treatment of hepatocellular carcinoma: expert consensus statement. HPB (Oxford). 2010;12(5):302-310.

37. Tiong L, Maddern GJ. Systematic review and meta-analysis of survival and disease recurrence after radiofrequency ablation for hepatocellular carcinoma. Br J Surg. 2011;98(9):1210-1224.

38. Desiderio J, Trastulli S, Pasquale R, et al. Could radiofrequency ablation replace liver resection for small hepatocellular carcinoma in patients with compensated cirrhosis? A 5-year follow-up. Langenbecks Arch Surg. 2013;398(1):55-62.

39. Mittler J, McGillicuddy JW, Chavin KD. Laparoscopic liver resection in the treatment of hepatocellular carcinoma. Clin Liver Dis. 2011;15(2):371-384.

40. Araki K, Conrad C, Ogiso S, Kuwano H, Gayet B. Intraoperative ultrasonography of laparoscopic hepatectomy: key technique for safe liver transection. J Am Coll Surg. 2013;(13):10.

41. Long TC, Bac NH, Thuan ND, Dat LT, Viet DQ, Chuong LC. Laparoscopic liver resection: 5-year experience at a single center. Surg Endosc. 2014;28(3):796-802.

42. Reggiani P, Antonelli B, Rossi G. Robotic surgery of the liver: Italian experience and review of the literature. Ecancermedicalscience. 2013; 7:358.

43. Fan ST, Poon RT, Yeung C, et al. Outcome after partial hepatectomy for hepatocellular cancer within the Milan criteria. Br J Surg. 2011;98(9):1292-1300.

44. Lee YH, Hsu CY, Huang YH, et al. Alpha-fetoprotein-to-total tumor volume ratio predicts post-operative tumor recurrence in hepatocellular carcinoma. J Gastrointest Surg. 2013;17(4):730-738.

45. Nagano H, Kobayashi S, Marubashi S, et al. Combined IFN-alpha and 5-FU treatment as a postoperative adjuvant following surgery for hepatocellular carcinoma with portal venous tumor thrombus. Exp Ther Med. 2013;5(1):3-10.

46. Riaz IB, Riaz H, Riaz T, et al. Role of vitamin $\mathrm{K} 2$ in preventing the recurrence of hepatocellular carcinoma after curative treatment: a meta-analysis of randomized controlled trials. BMC Gastroenterol. 2012;12:170.

47. Kishi Y, Hasegawa K, Sugawara Y, Kokudo N. Hepatocellular carcinoma: current management and future development-improved outcomes with surgical resection. Int J Hepatol. 2011;2011:728103.

48. Liu F, Wei Y, Wang W, et al. Salvage liver transplantation for recurrent hepatocellular carcinoma within UCSF criteria after liver resection. PLoS One. 2012;7(11):e48932. 